\title{
河南灵井许昌人遗址大型食草类动物死亡年龄分析 及东亚现代人类行为的早期出现
}

张双权 ${ }^{\circledR Q}$, 李占扬 ${ }^{(3)}$, 张乐 ${ }^{(Q)}$, 高星 ${ }^{(囚 *}$

(1) 中国科学院古脊椎动物与古人类研究所, 北京 100044 ;

(2) 中国科学院人类演化与科技考古联合实验室, 北京 100044;

(3) 河南省文物考古研究所, 郑州 450000

* 联系人, E-mail: gaoxing@ivpp.ac.cn

2009-07-08 收稿, 2009-09-03 接受

国家重点基础研究发展计划(编号: 2006CB806400)和国家自然科学基金(批准号: 40602006, 40872115)资助项目

摘要 以近期发现的旧石器时代中期的河南灵井许昌人遗址动物群为主要研究目标. 埋藏 学的分析表明, 原始牛和普通马是该动物群的优势属种, 同时也是灵井许昌人时期古人类 的主要猎捕对象. 在死亡年龄分布模式方面, 这两种大型食草类动物都是以壮年个体占据 绝对数量优势的一种组合面貌, 说明这一时期的东亚古人类已经是十分熟练的狩猎者, 他 们完全有能力猎捕原始牛、普通马等大型食草类动物. 这一研究结果表明, 东亚旧石器时 代中期及晚期的古人类在狩猎策略、能力以及群体组织等方面已经不存在明显差异, 从而

关键词

现代人类行为起源 旧石器时代中期 灵井许昌人遗址 埋藏学

动物考古学 验证了这一地区现代人类行为起源的早期理论.

现代人类行为的起源问题是旧石器时代考古学 研究中的一个争论热点. Klein 等人 ${ }^{[1 \sim 3]}$ 以及其他学者 认为, 现代人类行为起源于非洲, 并且这一行为的出 现是一个相对快速的“革命性”的过程, 大约是在距今 50000 40000 年之间; McBrearty 等人 ${ }^{[4]}$ 及 d'Errico ${ }^{[5]}$ 则认为, 现代人类行为的出现应该远远早于这一时间; 而且, 这一行为的最早出现可能并非仅仅局限于非洲, 近东甚至包括欧洲都有可能是其发源之地 ${ }^{[5]}$.

专业化的狩猎能力是现代人类行为出现的一个 重要标志 ${ }^{[4,6]}$. 从 20 世纪 80 年代初期至今, 古人类狩 猎能力的成熟与否一直是旧石器时代中期(MP)以及 与其大致相当的石器时代中期(MSA)考古动物群研 究中的主要问题. Binford 等人 ${ }^{[710]}$ 根据非洲以及欧亚 大陆 MSA/MP 时期动物群的骨骼单元分布模式、食 肉类的破坏痕迹、死亡年龄分布等方面的证据认为,
这一时期的古人类在大中型动物资源的利用方面是 以食腐作为其主要生存手段和策略的; 狩猎活动只 是古人类在对付一些中小型食草类动物时才能够采 用的一种生存手段. Binford等人 ${ }^{[7 \sim 10]}$ 的这一假说受到 了来自以 Marean 等人 ${ }^{[11,12]}$ 为代表的一大批学者的强 力批评. 最近一段时间, 在 MSA/MP 时期古人类的狩 猎食腐行为争论之外, 学者们又给这一阶段的动物 群研究赋予了新的学科张力, 从而也催生了新一轮

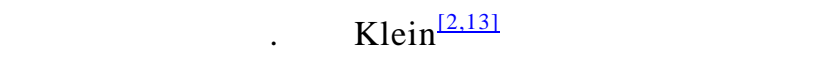
学者认为, MSA/MP 时期的古人类已经能够猎捕一些 有蹄类动物, 但是, 他们的狩猎技能却明显要劣于石 器时代晚期(LSA)/旧石器时代晚期(UP)阶段的古人 类 Klein 认为, 发生在距今大约 50000 40000 年左右 的一次古人类神经系统的重组或变异使古人类具备 了真正意义上的现代人的智能水平; 古人类在智能 
方面的提升又催生了更为有效的狩猎方式的出现以 及随后发生的现代人类的全球性扩散 ${ }^{[1,2,13]}$. 在 Klein 之外的另外一些学者, 却坚持认为这一时期的古人 类已经是非常熟练的狩猎者, 现代狩猎行为的出现 也只是 MSA/MP 时期出现的一系列现代人类行为标 志中的一个; 至于现代人类向欧亚大陆的扩散则可 以归因于古人类在技术发展、社会活动性乃至人口扩 张等方面的原因 ${ }^{[4,14]}$.

遗憾的是，在这几波现代人类行为之争的浪潮 中, 目前几乎所有的声音都来自于非洲、欧洲以及近 东地区; 相反, 东亚地区的考古动物群材料却总是处 于一种相对缄言的状态, 没有得到国际学术界的足 够关注与重视 ${ }^{[15]}$. 灵井许昌人遗址考古材料的近期 出土是我国古人类旧石器研究领域内的一个重要发 现, 同时也为东亚地区现代人类行为起源问题的研 究提供了良好的契机. 到目前为止, 该遗址出土的人 类骨骼材料包括顶骨、枕骨部分、乳突等近 20 件; 与 古人类头骨化石伴生的则是数量可观的旧石器文化 遗物及动物化石材料. 李占扬等人 ${ }^{[16]}$ 根据生物地层 学的原则判断, 这一动物群的时代应与许家窑动物 群的时代相似, 为晚更新世早期, 其绝对年龄约在 $100 \mathrm{ka}$ 左右. 周立平等人完成的初步光释光测年结果 表明，埋藏古人类头骨化石层位的时代应该在 80 100 ka 之内, 甚至可能稍大于 10 万年(详细成果 将另文发表). 这一时间段是探讨东亚地区现代人类 行为起源时间与地点的一个关键节点.

\section{1 材料与方法}

灵井许昌人遗址位于河南省许昌市西北约 15 $\mathrm{km}$ 的灵井镇西侧. 2005 2009 年, 河南省文物考古研 究所对该遗址进行了新的发掘与研究, 在近 $300 \mathrm{~m}^{2}$ 的范围内出土人类头骨化石断块近 20 件、石制品近 万件以及包括部分骨器在内的动物骨骼化石万余件. 到目前为止, 灵井许昌人遗址出土的哺乳动物化石 种类包括啮齿类 2 种, 食肉类 3 种, 长鼻类 1 种, 奇 蹄类 4 种，偶蹄类 8 种，共计 18 个属种 ${ }^{[16]}$. 灵井动物 群的埋藏学分析表明, 原始牛(Bos primigenius)和普 通马 (Equus caballus)是该动物群中居于绝对数量优 势的两个属种(图 1) $)^{[17]}$.

在考古动物群的研究中, 判定动物的死亡年龄 或死亡季节对于遗址埋藏学历史的重建具有非常重 要的意义 ${ }^{[18,19]}$; 同时, 通过此类研究, 我们还有可能

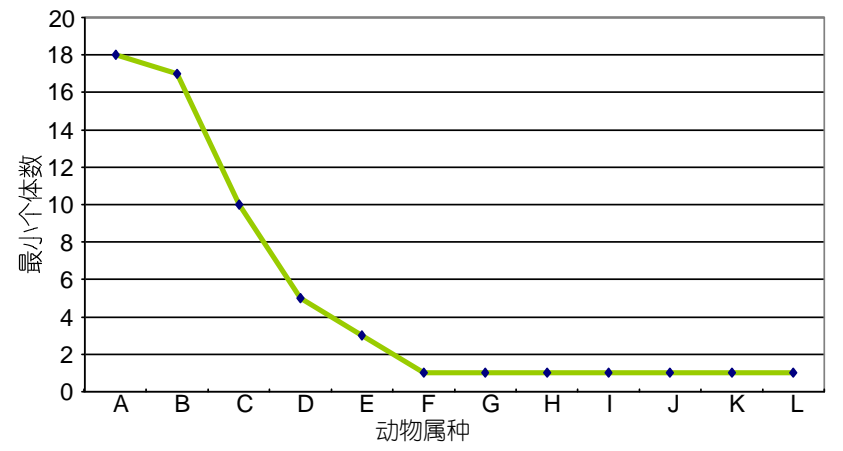

图 1 灵井动物群主要动物属种的最小个体数分布

A, Equus sp.; B, Bos primigenius; C, Megaloceros ordosianus (Cervus elaphus); D, Coelodonta antiquitatis; E, Procapra przewalskii; F, Dicerorhinus mercki; G, Pachycrocuta cf. sinensis; H, Palaeoloxodon sp.; I, Viverra cf. zibetha; J, Ursus sp.; K, Sus lydekkeri; L, Hydropotes pleistocenica

进一步提取古人类行为能力、社会组织、群体规模乃 至狩猎方式、技能、生存模式等方面的重要信息. 通 常情况下，可以判断动物死亡年龄及季节的主要有 3 种方法：骨骺愈合、牙齿严质的年轮以及齿冠高度等 (牙齿冠面的磨蚀特征也是许多研究后期动物群的考 古学家经常使用的一种方法，但是对于更为早期的 动物群而言, 似乎这一手段的应用远远没有其他方 法那样普遍).一般情况下, 对于大型有蹄类动物而 言，由于骨䯚愈合和牙齿严质分析方面的诸多困难， 齿冠高度的测量和统计目前依然是埋藏学家确定动 物死亡年龄时最常采用的一种方法. 在本项研究中, 我们依据埋藏学研究的经典做法分别测量了灵井动 物群中的两种优势动物——原始牛和普通马的齿冠 高度, 并根据相应的回归方程计算得出了这些牙齿 标本所代表的动物个体的死亡年龄 18220$]$. 在各动物 个体的年龄确定之后，我们遵循 Klein 等学者的传统 做法, 将其归类并人各年龄组别(这里, 我们采取的 是 6 阶段的划分方案), 并以直方图的形式较为直观 地表示了各年龄段的个体数量差异.

在原始牛和普通马死亡年龄的研究过程中, 我 们同时也采用了 Stiner 所创立的动物年龄分组方案. Stiner ${ }^{[9,10,21]}$ 将动物个体按照其年龄差别分成了 3 个阶 段，即幼年、壮年和老年，这是目前国际动物考古学 界较为广泛采用的一种规范化手段 ${ }^{[22,23]}$. 我们之所以 采用这一分组体系，一方面是由于它与动物个体的 生命史相互吻合，从而可以较为合理地反映古人类 在狩猎趋向、能力方面的一些时空变化; 同时, 由于 20 世纪 90 年代以后的绝大多数考古动物群研究都把 
这一分组体系纳入了动物死亡年龄模式的构建方面, 因此这一方法客观上也增加了不同动物群之间以及 多个动物属种之间进行对比研究的可能性.

在 Stiner 的这一分组方案中, 某一动物属种幼年 与壮年的界限对应于动物个体的某一乳齿系牙齿被 其相应位置的恒齿系牙齿所替代的年龄(如 $\mathrm{dp} 4$ 和 $\mathrm{p} 4$ ); 壮年与老年的界限则大约是在该物种最大寿命值的 $65 \%$ 左右 ${ }^{[10,21]}$. 这里, 我们将原始牛幼年与壮年的界 限定义为 4 岁; 由于原始牛的理论最大寿命一般多被 界定为 25 岁 ${ }^{[24]}$ ，因此其壮年与老年的分界线年龄应 在 15 岁左右. 对于普通马而言, 它们的 p2一般在 4 岁之前已经完全萌出并开始接受磨蚀, 而其 $\mathrm{m} 3$ 的相 应年龄一般则在 2.5 5 岁左右 ${ }^{[25]}$; 结合现代马骨骺愈 合年龄的有关数据 ${ }^{[26]}$, 我们这里以 4 岁作为普通马幼 年个体的上限年龄; 同时, 我们依据 Levine $\mathrm{e}^{[26]}$ 的研究 结论以 17 岁作为其壮年与老年的界限值.

需要特别说明的是, 无论是在 Klein 还是 Stiner 的分类体系中, 用来构建动物死亡年龄图式的基本 统计单元更多情况下都是最小个体数(MNI), 其所依 据的牙齿材料都是某一侧的 $\mathrm{dp} 4$ 和 $\mathrm{m} 3$ (或者是 $\mathrm{dp} 4$ 和 $\mathrm{p} 4$ ). 但是, 在本次灵井动物群的研究方面, 我们
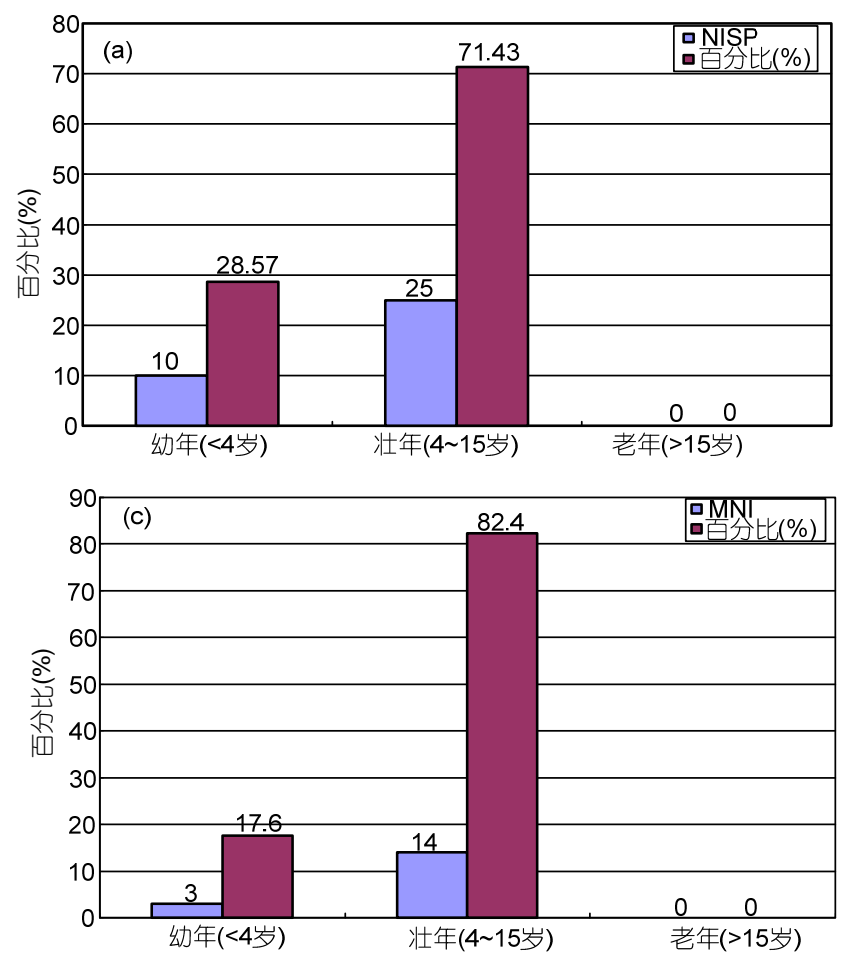

却采取了一些有别于传统做法的调整性方案. 以原 始牛为例, 其最小个体数仅为 17 个, 样本规模稍显 偏小一些, 并不十分有利于统计分析的进行. 出于这 一考虑, 我们在保留传统研究方法的同时也适当增 加了用于统计分析的动物牙齿材料. 这一调整明显 扩大了用于动物死亡年龄统计的样本规模, 从而也 较为有效地避免了由此而带来的可能偏差. 实际上, 在考古动物群的研究中, 欧洲的部分学者同样采用 了这一手段以规避因样本规模大小而带来的一些问 题 ${ }^{[18,19,27]}$; 在周口店田园洞梅花鹿化石的年龄结构分 析方面, 李青和同号文 ${ }^{[28]}$ 采用的也是这一处理方案.

\section{2 结果}

\section{1 原始牛的死亡年龄分布模式}

灵井动物群中原始牛个体的死亡年龄分析结果 表明, 无论是在 Stiner 的三分体系中还是在我们相对 细化的 6 阶段划分方案中, 无论是以最小个体数 (MNI)作为统计单元还是以包含更多齿列标本的可鉴 定标本数(NISP)作为统计基数, 这一动物属种都呈现 出了一种相当典型的“壮年居优型”的死亡年龄分布 模式 ${ }^{[29]}$ (图 2).
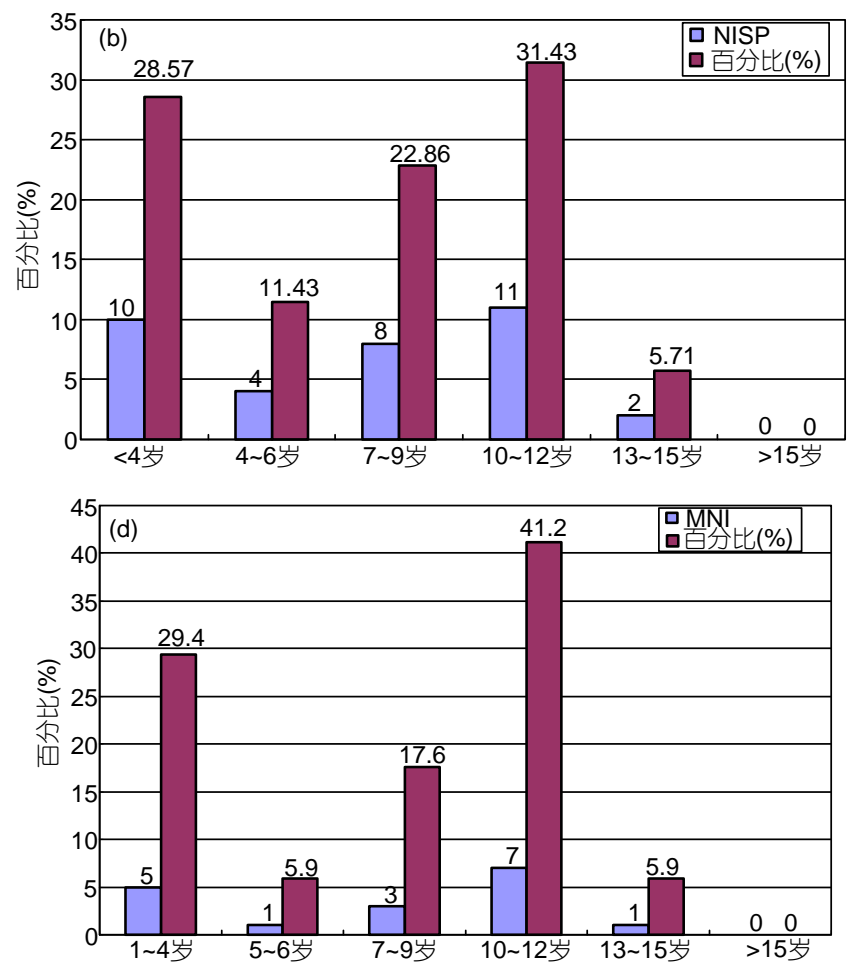

图 2 灵井动物群原始牛的死亡年龄分布

(a) NISP-3 年龄组; (b) NISP-6 年龄组; (c) MNI-3 年龄组; (d) MNI-6 年龄组 


\section{2 普通马的死亡年龄分布模式}

灵井动物群中普通马个体的死亡年龄分析结果 表明(这里我们仅以最小个体数的三阶段分组方案予 以说明)：与此前我们看到的原始牛的分布模式基本 类似，普通马的死亡年龄分布模式同样是以壮年个 体占有绝对优势，幼年个体则相对较少，而老年个体 更是只有 1 例, 仅占最小个体数的 $5.6 \%$ (图 3).

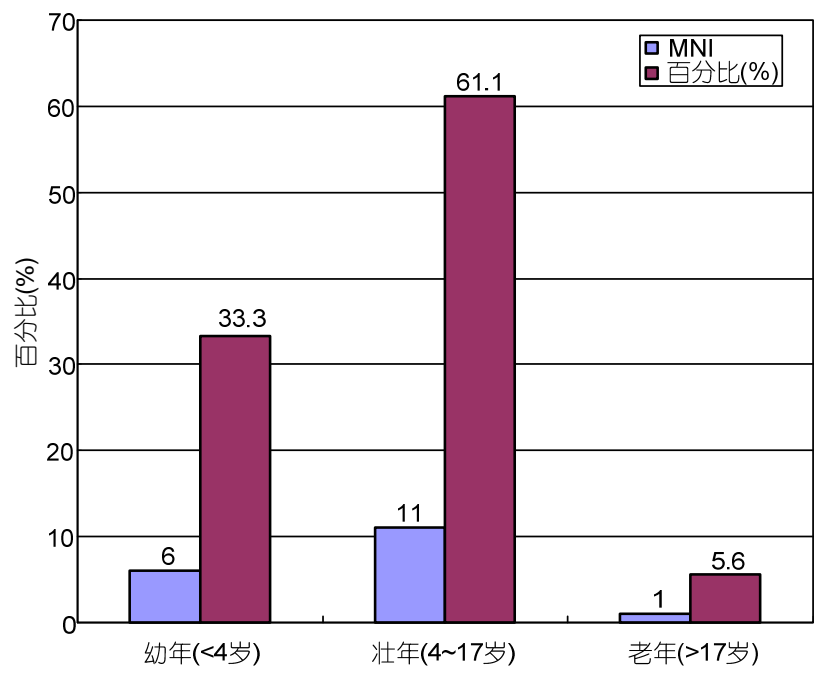

图 3 灵井动物群普通马的死亡年龄分布(MNI-3 年龄组)

\section{3 灵井许昌人遗址原始牛死亡年龄结构与相关动} 物群的比较

在旧石器时代中期的许多遗址中，包括欧洲的 法国、意大利、德国以及近东的以色列等国家都曾经 发现了古人类狩猎和屠宰原始牛或野牛的证据. 与 灵井动物群极为相似的是, 这些遗址中的许多动物 种类都呈现出了一种以壮年个体占据绝对数量优势 的死亡年龄结构. 这其中就有我们比较熟知的法国 的 La Borde, Mauran 遗址、前苏联境内的 Il'skaya 遗 址、以色列的 Hayonim, Kebara 两个洞穴遗址以及意 大利的 Bruil 遗址等. 此外, 在欧洲旧石器时代晚期 的 Palidoro, Polesini 等几个遗址以及美国历史时期的 Agate Basin, Casper, Lamb Spring Site 和 Garnsey 等遗 址也都是以原始牛或野牛的壮年个体占据绝对数量 优势的一种死亡年龄组合 30,31$]$.

这里, 我们认为特别值得一提的就是法国的 $\mathrm{La}$ Borde 遗址. 与灵井许昌人遗址近似, La Borde 也是 一处旧石器时代中期的旷野遗址. 这一遗址中的动 物化石材料无论是从可鉴定标本数(NISP), 还是最小
个体数 $(\mathrm{MNI})$ 来看都以原始牛占据其绝对优势地位; 此外, 在 27 个原始牛个体中, 壮年个体占到了其中 的 $76.4 \%$, 幼年个体占 $21.6 \%$ ，老年个体仅为 $2 \%$ 左 右 ${ }^{[31]}$. 尽管这一动物群的骨骼表面保存情况不佳, 无 法进行切割痕、砍砸痕以及食肉类齿痕的分析统计, 但是较为系统的原始牛死亡年龄结构的研究还是为 这一遗址的考古学解释奠定了良好的基础. 研究者将 这一遗址最终解释为古人类的一个狩猎营地, 古人类 在此以原始牛的“育儿群(Nursery group)”为其狩猎对 象，从而导致了这些动物骨骼的大量聚集 ${ }^{[31]}$.

从灵井动物群原始牛与上述多个遗址类似动物 属种的年龄比较来看, 它们基本上都落在了“壮年居 优型”的年龄分类范围内. 也就是说, 与这些已经得 到广泛认可的动物群一样, 灵井动物群反映了旧石 器时代中期古人类的相对成熟而系统的生存活动和 社会组织形式(图 4).

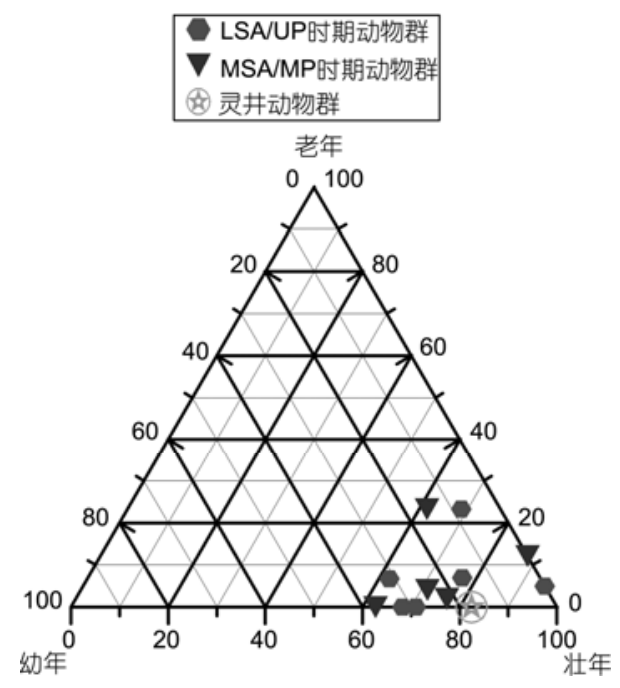

图 4 灵井动物群原始牛与相关动物群的死亡年龄 分布模式对比

3 讨论

3.1 动物死亡年龄分布模式中的埋藏学因素

灵井动物群中的原始牛、普通马两种动物都是以 壮年个体占据数量优势, 同时又有一定比例幼年成 员的一种死亡年龄组合. 这种以壮年成员为主要代 表类群的动物死亡模式在考古动物群的研究上具有 非常特殊的意义. Stiner ${ }^{[10,21]}$ 认为, 这一模式是古人类 行为的独特体现，反映了古人类在狩猎活动中的选 择性倾向; 西方许多学者也将这一现象与古人类的 
生存能力、狩猎方式以及现代人类行为的出现等问题 联系在了一起 ${ }^{[30-32]}$.

然而, 与此同时, 也有学者开始从现代生态观察 以及实验研究、埋藏学分析等多种角度质疑这一年龄 分布模式的合理性. 事实上, 埋藏学的研究已经表明, 考古动物群中出现的相对较少的幼年个体有时也许 并非是古人类的行为所致, 因为包括沉积成岩作用 在内的许多埋藏学过程都有可能造成某些动物属种 的幼年牙齿标本的大量减少和消失 ${ }^{[33]}$. 此外, 对于许 多考古遗址而言, 由于大型食肉类动物的后期破坏 作用, 许多幼年动物个体的骨骼单元, 有时也包括它 们的牙齿, 同样很难完整保留下来. 也就是说, 许多 情况下, 我们最终发现的也许只是一个严重“扭曲” 了的动物群年龄组成 ${ }^{[34,35]}$.

对于灵井许昌人遗址而言，尽管我们目前还无 法完全排除食肉类以及水流作用、沉积成岩作用等埋 藏学过程对于动物群面貌的改造, 但是, 灵井动物群 中保存良好的骨骼表面状况、极低比例的水流磨蚀标 本数量以及均一而极端弱化的骨骼风化程度无不说 明在动物骨骼沉积之后的水动力作用、风化作用等破 坏性的埋藏学过程对于这一动物群的影响是较为有 限的; 同时, 在骨骼的沉积成岩过程中, 依然没有特 别明显的破坏性因素的出现和作用, 证明了灵井动 物群形成过程中相对较弱的原地破坏作用的存在 ${ }^{[17]}$.

大中型食肉类动物能够造成中小型食草类动物 (如山羊和绵羊等)乳齿系标本的大量缺失 ${ }^{[36]}$; 但是, 对于牛、长颈鹿等大型有蹄类动物而言, 即便是瓿狗 这样的最为强悍的骨骼破坏者, 往往也很难导致这 些动物牙齿标本数量的大幅锐减 ${ }^{[37]}$. 同时, 灵井动物 群中低至 5.4\%的齿痕比例及其分布特点也强烈暗示 了相对较为微弱的食肉类活动的影响; 人工切割痕 的分布规律及组合特点进一步验证了古人类在这一 动物群形成过程中的主导性作用 ${ }^{[17]}$.

综上所述, 我们相信, 灵井动物群原始牛、普通 马的年龄结构很少受到后期埋藏学过程的影响; 这 一动物群的当前面貌基本应是其原始死亡群的真实 体现. 因此, 我们对于这些动物属种年龄结构的考古 学分析和研究应该是较为客观和真实的.
3.2 “壮年居优死亡模式”的考古学意义

“壮年居优死亡模式”是晚更新世和全新世考古 动物群中非常典型的一种年龄结构 ${ }^{[10,21,38]}$; 欧亚大陆 MP 时期的动物群记录中同样不乏类似的记录 ${ }^{[30,31]}$. 民族学及民族考古学的证据将 “壮年居优死亡模式” 与人类的潜伏式狩猎往往联系在了一起, 而不论狩 猎人群的规模大小 ${ }^{[38]}$.

长期以来, 动物考古学家都把原始牛和非洲水 牛,尤其是其成年个体视为是极端危险而难以猎捕的 动物 ${ }^{[2,39,40]}$. “壮年居优死亡模式”在灵井动物群原始 牛以及普通马化石材料中的出现表明: 这一时期的 古人类已经掌握了足够的狩猎知识与技能; 他们对 于周边的自然环境也已有了非常深入的了解和适应. 尤其是在和大型食草类动物的关系方面, 古人类显 然已经熟知了这些“邻居”的生活习性及迁徙规律, 因此他们总是能够适时实地的调整自己的狩猎方式 和生存策略, 从而确保他们可以经常性地猎取到性 情极度凶猛的原始牛和普通马的壮年个体.

\section{4 结语}

目前, 已有越来越多的考古材料表明，欧亚大陆 旧石器时代中期与晚期的古人类都已经能够经常性 地狩猎某些大中型的食草类动物, 而食腐行为只是 偶尔伴随在古人类主流狩猎活动身边的一种随机现 象 ${ }^{[4,40]}$. 同样, 在狩猎技能方面, 这两个时期的古人 类也都能够熟练地猎捕如原始牛、野牛之类的凶猛的 大型食草类动物 ${ }^{[14,22,30,31,38]}$, 甚至还包括体型更为庞 大的猛犸象和犀牛 ${ }^{[41,42] ;}$ 此外, 在社会组织和专业化 的狩猎行为方面, 这两个时期的古人类几乎也已是 别无二致：他们不仅能够通过相关的社会性活动组 织群体的狩猎行为; 而且, 在现实需要的情况下, 他 们也都能够集中于某一种或两种猎物或是其中某些 特定年龄或性别的个体, 从而实现专业化的狩猎策 略. 灵井许昌人遗址原始牛和普通马的化石材料不 仅印证了这一时期古人类成熟而系统的狩猎能力和 群体组织行为, 同时也为东亚地区现代人类行为起 源的早期理论提供了十分重要的考古学证据. 


\section{参考文献}

1. Klein R G. The Human Career. 2nd ed. Chicago: University of Chicago Press, 1999

2. Klein R G. Archeology and the evolution of human behavior. Evol Anthropol, 2000, 8: 17-36[doi]

3. Mellars P. The impossible coincidence. A single-species model for the origins of modern human behavior in Europe. Evol Anthropol, 2005, 14: 12-27[doi]

4. McBrearty S, Brooks A S. The revolution that wasn't: A new interpretation of the origin of modern human behavior. J Hum Evol, 2000, 39: 453-563리이

5. d'Errico F. The invisible frontier. A multiple species model for the origin of behavioral modernity. Evol Anthropol, 2003, 12: 188 - 202[doi]

6. Henshilwood C S, Marean C W. The origin of modern human behavior: Critique of the models and their test implications. Curr Anthropol, 2003, 44: 627-651[doi]

7. Binford L R. Bones: Ancient Men and Modern Myths. New York: Academic Press, 1981

8. Binford L R. Faunal Remains from Klasies River Mouth. New York: Academic Press, 1984

9. Stiner M C. The use of mortality patterns in archaeological studies of hominid predatory adaptations. J Anthropol Archaeol, 1990, 9: $305-351$ [doi]

10. Stiner M C. Honor Among Thieves. Princeton: Princeton University Press, 1994

11. Marean C W, Kim S Y. Mousterian large-mammal remains from Kobeh Cave: Behavioral implications for Neanderthals and early modern humans. Curr Anthropol, 1998, 39: 79-113[doi]

12. Thompson J. Zooarchaeological tests for modern human behavior at Blombos Cave and Pinnacle Point Cave 13B, Southwestern Cape, South Africa. Ph.D Dissertation. Arizona: Arizona State University, 2008

13. Klein R G. Out of Africa and the evolution of human behavior. Evol Anthropol, 2008, 17: 267—281 [doi]

14. Faith J T. Eland, buffalo, and wild pigs: Were Middle Stone Age humans ineffective hunters? J Archaeol Sci, 2008, 55: 24-36

15. Norton C J, Gao X. Hominin-carnivore interactions during the Chinese Early Paleolithic: Taphonomic perspectives from Xujiayao. J Hum Evol, 2008, 55: 164-178 [doi]

16. 李占扬, 董为. 河南许昌灵井旧石器遗址哺乳动物群的性质及时代探讨. 人类学学报, 2007, 26: 345一 360

17. 张双权. 河南许昌灵井动物群的埋藏学研究. 博士学位论文. 北京: 中国科学院研究生院, 2009. 1-216

18. Fernandez P, Legendre S. Mortality curves for horses from the Middle Palaeolithic site of Bau de l'Aubesier (Vaucluse, France): Methodological, palaeo-ethnological, and palaeoecological approaches. J Archaeol Sci, 2003, 30: 1577-1598[doi]

19. Fernandez P, Jean-Luc G, Philippe F. Applying dynamics and comparing life tables for Pleistocene Equidae in anthropic (Bau de l'Aubesier, Combe-Grenal) and carnivore (Fouvent) contexts with modern feral horse populations (Akagera, Pryor Mountain). J Archaeol Sci, 2006, 33: 176-184 [doi]

20. Klein R G, Cruz-Uribe K. The Analysis of Animal Bones from Archaeological Sites. Chicago: University of Chicago Press, 1984

21. Stiner M C. The Faunas of Hayonim Cave (Israel): A 200000-Year Record of Paleolithic Diet, Demography and Society. Cambridge: Peabody Museum Press, 2005

22. Adler D S, Bar-Oz G, Belfer-Cohen A, et al. Ahead of the game: Middle and Upper Palaeolithic hunting behaviors in the southern Caucasus. Curr Anthropol, 2006, 47: 89-118 $\underline{\text { [doi] }}$

23. Bar-Oz G, Adler D S. Taphonomic history of the Middle and Upper Palaeolithic faunal assemblages from Ortvale Klde, Georgian Republic. J Taphonomy, 2005, 3: 185-211

24. Gifford-Gonzalez D. Examining and refining the quadratic crown height method of age estimation. In: Stiner M C, ed. Human Predation and Prey Mortality. Boulder: Westview Press, 1991. 41-78

25. Hillson S. Teeth. 2nd ed. Cambridge: Cambridge University Press, 2005

26. Levine M A. Archaeo-zoological analysis of some Upper Pleistocene horse bone assemblages in Western Europe. Ph.D Dissertation. Cambridge: University of Cambridge, 1979

27. Enloe J G, Turner E. Methodological problems and biases in age determinations: A view from the Magdalenian. In: Ruscillo D, ed. Recent Advances in Ageing and Sexing Animal Bones: 9th ICAZ Conference, Durham 2002. Oxford: Oxbow Press, 2006. 129—144

28. 李青, 同号文. 周口店田园洞梅花鹿年龄结构分析. 人类学学报, 2008, 27: 143-152

29. Norton C J, 张双权, 张乐, 等. 上/更新世动物群中人类与食肉动物“印记”的辨识. 人类学学报, 2007, 26: 183一192

30. Gaudzinski. On bovid assemblages and their consequences for the knowledge of subsistence patterns in the Middle Palaeolithic. P Prehist Soc, 1996, 62: 19-39

31. Jaubert J, Lorblanchet M, Laville H, et al. The Hunters of Aurochs of La Borde (in French). Documents of French Archaeology. Paris: The Maison of Sciences of Humans, 1990

32. Steele T E. Red deer: Their ecology and how they were hunted by Late Pleistocene hominids in Western Europe. Ph.D Dissertation. 
Stanford: Stanford University, 2002

33. Lyman R L. Vertebrate Taphonomy. Cambridge: Cambridge University Press, 1994

34. Marean C W, Blumenschine R J. Captive hyaena bone choice and destruction, the schlepp effect and Olduvai archaeofaunas. J Archaeol Sci, 1992, 19: 101-121 $\underline{\text { [doi] }}$

35. Munson P J, Marean C W. Adults only? A reconsideration of Middle Paleolithic "prime-dominated" reindeer hunting at Salzgitter Lebenstedt. J Hum Evol, 2003, 44: 263-273[doi]

36. Munson P J. Age-correlated differential destruction of bones and its effect on archaeological mortality profiles of domestic sheep and goats. J Archaeol Sci, 2000, 27: 391-407 [doi]

37. Bunn H T, Kroll E M. Systematic butchery by Plio-Pleistocene hominids at Olduvai Gorge, Tanzania. Curr Anthropol, 1986, 27: $431-452$ [doi]

38. Bar-Oz G. Epipaleolithic Subsistence Strategies in the Levant: A Zooarchaeological Perspective. Boston: Brill Academic Publishers, 2004

39. Speth J D, Clark J. Hunting and overhunting in the Levantine late Middle Palaeolithic. Before Farm, 2006, 3: 1-42

40. Speth J D, Tchernov E. Neandertal hunting and meat-processing in the near East: Evidence from Kebara Cave (Israel). In: Stanford C B, Bunn H T, eds. Meat-Eating and Human Evolution. Oxford: Oxford University Press, 2001. 52-72

41. Callow P, Cornford J. La Cotte de St. Brelade. Norwich, UK: Geo Books, 1986

42. Villa P, Lenoir M. Hunting weapons of the Middle Stone Age and the Middle Palaeolithic: Spear points from Sibudu, Rose Cottage and Bouheben. South Afr Humanit, 2006, 18: 89-122

・动 态・

\section{周口店遗址新发掘有望破解基础科学问题}

2009 年 6 月 24 日, 中国科学院古脊椎动物与古人类研 究所的一支科考队携带着发掘工具、仪器出现在周口店遗 址第 1 地点西剖面上. 这是该遗址“北京人”生活过的地层 在发掘中断近半个世纪后又一次被考古的探铲触及，立刻 引起学术界和媒体的高度关注, Science 杂志对此做了专题 报道.

周口店是重要的古人类遗址，是珍贵的人类文化遗产. 1961 年被国务院公布为全国重点文物保护单位, 1987 年被 联合国教科文组织列入世界文化遗产名录. 第 1 地点是周 口店遗址中最重要的地点, 出土代表 40 余个个体的直立人 化石和大量石器、哺乳动物化石和用火遗迹, 在研究东亚 人类起源和直立人演化方面具有不可替代的地位和作用. 该地点西剖面是遗址的心脏, 保存有完整的地层序列和丰 富的材料与信息, 是考察研究的核心部位, 也是遗址保护 的重点对象.

这次发掘并非刻意寻找古人类化石和文化遗存, 而是 一次遗产抢救行动, 是为落实遗址保护规划而进行的保护 性发掘. 该遗址原为洞穴, 在古人类生存的后期坍塌; 1927 1937 年间大规模发掘清除了洞内的大部分沉积, 改 变了遗址的自然环境; 发掘后的剖面长期暴露, 在重力和风 化作用下变得凹凸不平, 出现松滑、孔洞、裂隙和危石, 呈 现上突下缩、头重脚轻的逆势坡度, 具有坍塌的隐患. 两个 月前, 西剖面上部(第 3 层)突现南北向开裂, 其下(第 4 层) 几近悬空, 随时有垮塌危险, 抢救发掘, 势在必行.

此次发掘在面积约 $10 \mathrm{~m}^{2}$ 、深度近 $30 \mathrm{~m}$ 的范围内进行.
发掘前对剖面进行了三维激光扫描测量，以保存、记录发 掘前的原始状态并为规划清理范围、进深和角度提供数据. 按照计划，至 2009 年 7 月下旬完成对顶部开裂部位的抢救 性发掘, 8 10 月实施对西剖面的整体清理和系统取样. 发 掘采用现代先进的田野考古理念与技术，以立体网格式探 方体系对地层和出土物进行布控，对出土的重要遗迹、遗 物用全站仪进行精准的三维坐标测量, 对出土的化石等标 本及时进行保护加固, 对地层中的所有土样进行篮拣, 并 采取水洗、水篮等方法提取微小的标本. 发掘时努力消除 地层堆积的各种隐患, 将剖面切割平整并调整出合理、稳定 的坡度; 对地层进行详细观测和系统取样, 以供沉积学、环 境学和年代学分析测试, 建立更加系统、精确的洞穴发育史 和年代、环境变迁框架.

此次发掘恰逢北京猿人第一头盖骨发现 80 周年. 80 年 间, 在对遗址发掘和研究不断取得重大学术收获的同时, 学术界对诸如“北京人”对洞穴的使用方式、用火和狩猎能 力、生存时代、环境背景以及遗址地层堆积过程和成因等 问题时有争议. 此次科考行动受到科技部重大基础性工作 专项基金的资助, 通过科学发掘、系统取样、多手段分析测 试和多学科交叉研究, 将获得丰富、系统、精细的资料和数 据, 夯实遗址研究的科学基础, 有望在上述重大而又有争议 的基础科学问题上取得创新和突破.

高星, 陈福友

(中国科学院古脊椎动物与古人类研究所, 北京 100044) 